\title{
A case study in bivariate singularity analysis
}

\author{
Timothy DeVries
}

\begin{abstract}
The multivariate singularity analysis of Pemantle and Wilson is explored and then used to derive an asymptotic expression for the number of bicolored supertrees, realized as the diagonal sequence of a bivariate rational function $F$. These asymptotics have been obtained previously by univariate methods, but the analysis contained herein serves as a case study for the general multivariate method. The analysis itself relies heavily on the structure of a height function $h$ along the pole set $\mathcal{V}$ of $F$. What makes this example interesting is the geometry of $h$ on $\mathcal{V}$, namely that $h$ has a degenerate saddle point away from the boundary of the domain of analyticity of $F$ that contributes to the asymptotic analysis. Due to the geometry of $h$ at this point, the coefficient analysis can not be computed directly from the standard formulas of multivariate singularity analysis. Performing the analysis in this case represents a first step towards understanding general cases of this geometric type.
\end{abstract}

\section{Introduction}

Let $F(x, y)=P(x, y) / Q(x, y)=\sum_{r, s \geq 0} a_{r, s} x^{r} y^{s}$ be the rational generating function defined by

$$
\begin{aligned}
& P(x, y)=2 x^{2} y\left(2 x^{5} y^{2}-3 x^{3} y+x+2 x^{2} y-1\right), \\
& Q(x, y)=x^{5} y^{2}+2 x^{2} y-2 x^{3} y+4 y+x-2 .
\end{aligned}
$$

In this paper we use multivariate singularity analysis to derive the asymptotic estimate

$$
a_{n, n} \sim \frac{4^{n}}{8 \Gamma(3 / 4) n^{5 / 4}} .
$$

While this result has been obtained previously by univariate methods, the multivariate analysis of this sequence proves to be interesting to the study of multivariate singularity analysis itself. Specifically, this example is interesting because it does not lend itself to automatic computation by the formulas set forth in [PW02], the main reference for the multivariate techniques we will use. In what follows, we briefly discuss how this affects the ultimate goal of producing automatic asymptotic analyses for multivariate rational generating functions. Before proceeding, however, it is important to discuss from where the function $F$ originates.

2000 Mathematics Subject Classification. Primary 05A16; Secondary 05-04, 13 P10.

Key words and phrases. asymptotics, bivariate, generating function, singularity, residue. 
The coefficients $a_{n, n}$ actually count bicolored supertrees, which are defined as follows. First, denote by $\mathcal{G}$ the class of Catalan trees, i.e. rooted, unlabelled, planar trees, counted by the number of nodes. The class $\mathcal{G}$ has generating function

$$
G(x)=\frac{1}{2}(1-\sqrt{1-4 x}),
$$

whose coefficients are the Catalan numbers. Denote by $\tilde{\mathcal{G}}$ the class of bicolorplanted Catalan trees: Catalan having an extra red or blue node attached to the root (likewise counted by the number of nodes). The class $\tilde{\mathcal{G}}$ has generating function

$$
\tilde{G}(x)=2 x G(x) .
$$

Then the class of bicolored supertrees, denoted by $\mathcal{K}$, is defined by the combinatorial substitution $\mathcal{K}=\mathcal{G} \circ \tilde{\mathcal{G}}$. That is, the elements of $\mathcal{K}$ are Catalan trees with each node replaced by bicolor-planted Catalan trees. The class $\mathcal{K}$ has algebraic generating function $K(x)=G(\tilde{G}(x))$. More explicitly,

$$
K(x)=\frac{1}{2}-\frac{1}{2} \sqrt{1-4 x+4 x \sqrt{1-4 x}}=2 x^{2}+2 x^{3}+8 x^{4}+18 x^{5}+64 x^{6}+O\left(x^{7}\right),
$$

with coefficients from [Slo09]. Denote by $k_{n}$ the coefficient of $x^{n}$ in the expansion of $K(x)$ above, i.e. the number of bicolored supertrees having $n$ nodes. An asymptotic estimate for the $k_{n}$ has been obtained by univariate analysis of $K(x)$ [FS09, examples VI.10 and VII.20].

As it turns out, $a_{n, n}=k_{n}$, and $F(x, y)$ was produced by Alex Raichev and Mark Wilson to have this exact property (see [RW08]). This was done using Safonov's algorithm, which is roughly a procedure for realizing multivariate algebraic generating functions as so-called diagonals of rational generating functions in one higher variable [Saf00]. The idea proposed by Raichev and Wilson was to use Safonov's algorithm to reduce asymptotic computations on algebraic generating functions to those on rational generating functions, albeit in one higher dimension. In the case of bicolored supertrees, however, the multivariate analysis is not straightforward, for reasons we presently discuss.

The technique of multivariate singularity analysis has the following basic structure: begin with Cauchy's Integral Formula, manipulate the integral/integrand, and end with saddle point integration. To be more explicit, let $F=\eta / Q$ be the generating function of the coefficients to be analyzed, with $\eta: \mathbb{C}^{d} \rightarrow \mathbb{C}$ entire and $Q \in \mathbb{Q}\left[x_{1}, \ldots, x_{d}\right]$. Cauchy's Integral Formula expresses the coefficients as an integral of a particular $d$-form. By appropriately adjusting this integral, we can rewrite this as the integral of a related $(d-1)$-form defined on the variety $\mathcal{V}_{Q}=\{\mathbf{x}: Q(\mathbf{x})=0\}$ along a cycle $C \subseteq \mathcal{V}_{Q}$. We define a height function $h$ on the variety $\mathcal{V}_{Q}$ related to the rate of decay of this new integrand. We then push this cycle down along $\mathcal{V}_{Q}$, minimizing the maximum of $h$ along $C$ at critical points of the function $h$. Under the right conditions, the coefficients can finally be approximated as saddle point integrals along $C$ in small neighborhoods of a finite set of these critical points, known as the contributing points. In the case $d=2$, all critical points of $h$ are actually saddle points of $h$, i.e. critical points of $h$ which are not local extrema.

This technique has been well studied (see [PW02] and [PW08]), to the point that bivariate rational generating function asymptotics can be computed automatically in many cases. Specifically when all the contributing points are minimal that is, on the generating function's boundary of convergence - then an explicit 
algorithm exists for determining which critical points contribute and computing the saddle point integral near these points (in the bivariate case). And when the generating function is combinatorial, i.e. when all its coefficients are non-negative, then the contributing points will all be minimal (under the standing assumption of [PW08], Assumption 3.6). In the case examined presently, however, the generating function is not combinatorial. Thus there is no guarantee that any contributing points will be minimal. In fact, we will show that the contributing point is not minimal.

Thus [PW02] does not provide us with the locations of the contributing points. Worse than that, however, is that even once the contributing points have been found, there is no formula automatically producing the correct saddle point computation in a neighborhood of these points. This is because the contour followed by $C$ in a neighborhood of the contributing points is not automatically known. (On the contrary, for minimal contributing points, an explicit path for $C$ near these points is known; see [PW02]). This is particularly bad when the contributing point is a degenerate saddle point for the height function. Since the height on $C$ is locally maximized at the contributing point, it must locally approach and depart along ascent and descent paths. A greater degree of degeneracy means more ascent/descent paths, hence more possibilities for the local path followed by $C$. And indeed in the case presently studied, the contributing point is a degenerate saddle point of the height function.

Understanding the saddle point integration near these degenerate saddles is particularly important because degenerate saddles arise frequently in combinatorial applications (despite the fact that they are nongeneric). A careful analysis of $[\mathbf{P W 0 2}]$ reveals that, in the absence of such degenerate saddles, one obtains leading term asymptotics only of the form $c A^{n} n^{p / 2}$ (for constants $c, A$ and integer $p$ ). By Safonov's algorithm, any univariate algebraic generating function can be realized as the diagonal of a bivariate rational generating function. But by univariate asymptotic methods, we know that the coefficients of such univariate functions can produce leading term asymptotics of the form $c A^{n} n^{p / q}$ for arbitrary $q \in \mathbb{N}$ (see [FS09, Section VII.7]), and so a multivariate analysis of the corresponding bivariate rational function should turn up a degenerate saddle whenever $q>2$.

Understanding this example represents a first step towards understanding what to do when the contributing saddle point is not on the boundary of convergence, and when the height function is a degenerate saddle at this point. The rest of this paper is concerned with examining this example. In Section 2 we will present the theory needed to reduce Cauchy's Integral Formula to a series of saddle point integrals, as outlined above. In Section 3 we will apply these methods to the function $F(x, y)$ defined earlier, obtaining our asymptotic estimate.

\section{Multivariate Singularity Analysis}

2.1. Coefficient Representation. For the duration of this paper, let $F$ : $\mathbb{C}^{d} \rightarrow \mathbb{C}$ be a function analytic in a neighborhood of the origin, having representation

$$
F(\mathbf{x})=\sum_{\mathbf{r} \in \mathbb{N}^{d}} a_{\mathbf{r}} \mathbf{x}^{\mathbf{r}}
$$


where $\mathbf{x}^{\mathbf{r}}$ is shorthand notation for $x_{1}^{r_{1}} \ldots x_{d}^{r_{d}}$. The goal is to obtain an asymptotic expansion for the coefficients $a_{\mathbf{r}}$ given $F$, and the main tool for this is Cauchy's Integral Formula.

TheOREM 2.1 (Cauchy's Integral Formula). Let $F$ be as above, analytic in a polydisc $D_{0}=\left\{\mathbf{x}:\left|x_{j}\right|<\varepsilon_{j} \forall j\right\}$, for some positive, real $\varepsilon_{j}$. Assume further that $F$ is continuous on the boundary torus $T_{0}=\partial D_{0}$, a product of loops around the origin in each coordinate, each one positively oriented with respect to the complex orientation of its respective plane. Then

$$
a_{\mathbf{r}}=\int_{T_{0}} \omega_{F}
$$

where

$$
\omega_{F}=\frac{1}{(2 \pi i)^{d}} \cdot \frac{F(\mathbf{x})}{x_{1} \cdot \ldots \cdot x_{d}} \mathbf{x}^{-\mathbf{r}} d \mathbf{x}
$$

Cauchy's Integral Formula can be found in most textbooks presenting complex analysis in a multivariable setting, and follows easily as an iterated form of the single variable formula. See, for example, [Sha92, p. 19].

We wish to use the structure of Cauchy's formula to obtain an asymptotic formula for $a_{\mathbf{r}}$ as $\mathbf{r} \rightarrow \infty$, but first we need to be more precise about what is meant by " $\mathbf{r} \rightarrow \infty$." There are many ways to send the vector $\mathbf{r}$ to infinity, but one of the most natural ways is to fix a direction in the positive $d$-hyperoctant and send $\mathbf{r}$ to infinity along this direction. Specifically, define the $(d-1)$-simplex $\Delta^{d-1}$ by

$$
\Delta^{d-1}=\left\{\left(\hat{r}_{1}, \ldots, \hat{r}_{d}\right): \hat{r}_{j} \geq 0 \forall j, \sum_{j=1}^{d} \hat{r}_{j}=d\right\},
$$

where we choose the convention that the $\hat{r}_{j}$ sum to $d$ for later notational convenience. Then any $\mathbf{r}$ in the positive $d$-hyperoctant can be written uniquely as $\mathbf{r}=|\mathbf{r}| \hat{\mathbf{r}}$, where $|\mathbf{r}| \in \mathbb{R}^{+}$and $\hat{\mathbf{r}} \in \Delta^{d-1}$. We examine $\mathbf{r}$ as $|\mathbf{r}| \rightarrow \infty$ and $\hat{\mathbf{r}} \rightarrow \hat{\mathbf{r}}_{0}$ for some fixed direction $\hat{\mathbf{r}}_{0} \in \Delta^{d-1}$.

Now we turn to the structure of the integrand $\omega_{F}$, specifically $\mathbf{x}^{-\mathbf{r}}$ (the portion that changes as we vary $\mathbf{r}$ ). With an eye on the end goal of reducing our computation to a saddle integral, we use the following representation (away from the coordinate axes):

$$
\mathbf{x}^{-\mathbf{r}}=\exp \left(-\sum_{j=1}^{d} r_{j} \ln x_{j}\right)=\exp \left(|\mathbf{r}| H_{\hat{\mathbf{r}}}(\mathbf{x})\right)
$$

where

$$
H_{\hat{\mathbf{r}}}(\mathbf{x})=-\sum_{j=1}^{d} \hat{r}_{j} \ln x_{j}
$$

When no confusion exists, we will simply refer to the function $H_{\hat{\mathbf{r}}}$ as $H$. Now the overall magnitude of the integrand will be an important factor in computing an asymptotic expansion for $a_{\mathbf{r}}$, and so we next examine the magnitude of $\exp \left(|\mathbf{r}| H_{\hat{\mathbf{r}}}(\mathbf{x})\right)$. We have

$$
\left|\exp \left(|\mathbf{r}| H_{\hat{\mathbf{r}}}(\mathbf{x})\right)\right|=\exp \left(|\mathbf{r}| \Re H_{\hat{\mathbf{r}}}(\mathbf{x})\right)=\exp \left(|\mathbf{r}| h_{\hat{\mathbf{r}}}(\mathbf{x})\right)
$$


where

$$
h_{\hat{\mathbf{r}}}(\mathbf{x})=\Re H_{\hat{\mathbf{r}}}=-\sum_{j=1}^{d} \hat{r}_{j} \ln \left|x_{j}\right| .
$$

When no confusion exists, we will simply refer to the function $h_{\hat{\mathbf{r}}}$ as $h$. The geometry of the height function $h$ will play an important role in our analysis.

As $|\mathbf{r}| \rightarrow \infty$, the above equations show that the magnitude of the integrand grows at an exponentially slower rate along points further away from the origin (where the height function $h$ is smaller). This motivates pushing the domain of integration out towards infinity, reducing the growth rate of the integrand on the domain over which it is integrated. Of course if $F$ has poles they will present an obstruction, but we can still try push the domain of integration around these poles. In the end we obtain an integral over two domains: one near the pole set of $F$ (obtained by pushing the original domain around the poles), and one past the pole set of $F$ (far away from the origin). This idea is formalized in the theorem below.

TheOREM 2.2. Let $F=P / Q$, with $P, Q: \mathbb{C}^{d} \rightarrow \mathbb{C}$ entire, where the vanishing set $\mathcal{V}_{Q}$ of $Q$ is smooth. Let $T_{0}$ be a torus as in Cauchy's Integral Formula. Let $T_{1} \subseteq \mathbb{C}^{d}$ be a torus homotopic to $T_{0}$ under a homotopy

$$
K: T \times[0,1] \rightarrow \mathbb{C}^{d}, \quad \text { with } T_{0}=T \times\{0\}, T_{1}=T \times\{1\},
$$

passing through $\mathcal{V}_{Q}$ transversely. Identifying $K$ with its image in $\mathbb{C}^{d}$, assume further that $K$ does not intersect the coordinate axes, and that $\partial K \cap \mathcal{V}_{Q}=\emptyset$. Define

$$
C=K \cap \mathcal{V}_{Q}
$$

Then for any tubular neighborhood $\nu$ of of $C$ in $K$, we have

$$
a_{\mathbf{r}}=\int_{T_{0}} \omega_{F}=\int_{\partial \nu} \omega_{F}+\int_{T_{1}} \omega_{F},
$$

given the proper orientation of $\partial \nu$.

Note: when we say $\mathcal{V}_{Q}$ is smooth we mean that $\mathcal{V}_{Q}$ has the structure of a smooth manifold (see [Bre93, p. 68]). And when we say that $K$ passes through $\mathcal{V}_{Q}$ transversely we mean that the image of $K$ intersects with $\mathcal{V}_{Q}$ transversely as (real) submanifolds of $\mathbb{C}^{d}$ (see [Bre93, p. 84]).

Proof. Counting (real) dimensions, $\operatorname{dim} \mathcal{V}_{Q}=2 d-2$ and $\operatorname{dim} K=d+1$. Hence their transverse intersection $C$ is a $d-1$ real-dimensional subspace of $K$.

Now take any tubular neighborhood $\nu$ of $C$ in $K$. As $\nu$ is a full-dimensional submanifold of the orientable manifold $K, \nu$ is orientable and hence its boundary $\partial \nu$ is orientable too. Given the proper orientation of $\partial \nu$, we have that

$$
\partial(K \backslash \nu)=T_{1}-T_{0}+\partial \nu
$$

Note that $\omega_{F}$ is holomorphic on $K \backslash \nu$. By Stokes' Theorem ([Bre93, p. 267]) and the fact that $\omega_{F}$ is an exact form we get

$$
\int_{T_{1}-T_{0}+\partial \nu} \omega_{F}=\int_{K \backslash \nu} d \omega_{F}=\int_{K \backslash \nu} 0=0,
$$

leading to the equality of the theorem. 
When $T_{1}$ is far enough away from the origin, $\int_{T_{1}} \omega_{F}$ is negligible (possibly even 0 ), and so the asymptotic analysis of the coefficients $a_{\mathbf{r}}$ reduces to an integral near the pole set of $F$. In the next section, we reduce this further to an integral on the pole set of $F$.

2.2. The Residue Theorem. In this subsection we present a theory generalizing the theory of residues of the complex analysis of one variable. The theory was developed by Jean Leray in 1959, and more details regarding the construction can be found in [AY83, Section 16]. The main result we obtain is Theorem 2.8 below, an analogue of the Cauchy Residue Theorem in one variable. Its application to coefficient analysis is found in Corollary 2.9 .

We restrict our attention to a limited part of Leray's theory, focusing on meromorphic $d$-forms in $\mathbb{C}^{d}$.

Definition 2.3. Let $\eta$ be a meromorphic $d$-form, represented as

$$
\eta=\frac{P}{Q} d \mathbf{x} \text { on a domain } U \subseteq \mathbb{C}^{d}
$$

where $P$ and $Q$ are holomorphic on $U$. Denote by $\mathcal{V}_{Q}$ the zero set of $Q$ on $U$, and assume that $\eta$ has a simple pole everywhere on $\mathcal{V}_{Q}$. Denote by $\iota: \mathcal{V}_{Q} \rightarrow U$ the inclusion map. Then we define the residue of $\eta$ on $\mathcal{V}_{Q}$ by

$$
\operatorname{Res}(\eta)=\iota^{*} \theta
$$

where $\iota^{*}$ denotes pullback by $\iota$ (see [Bre93, p. 263]), and where $\theta$ is any solution to

$$
d Q \wedge \theta=P d \mathbf{x}
$$

Before delving into the existence and uniqueness of the residue, we do a few example computations.

ExAmple 2.4. For $\eta=P / Q d \mathbf{x}$ as above, wherever $Q_{i}=\frac{\partial Q}{\partial x_{i}}$ does not vanish we have the representation

$$
\operatorname{Res}(\eta)=(-1)^{i-1} \frac{P}{Q_{i}} d x_{1} \wedge \cdots \wedge d x_{i-1} \wedge d x_{i+1} \wedge \cdots \wedge d x_{d}
$$

As a special case, note that for $Q=x_{1}$ we obtain

$$
\operatorname{Res}(\eta)=P\left(0, x_{2}, \ldots, x_{d}\right) d x_{2} \wedge \cdots \wedge d x_{d} .
$$

In the case where $d=1$, this reduces to $\operatorname{Res}(P(x) / x)=P(0)$, which is precisely the ordinary residue of $P(x) / x$ at $x=0$. This motivates the above definition as a genuine extension of the single variable residue.

EXAMPLE 2.5. As the most pertinent case of the Example 2.4, we examine $\operatorname{Res}\left(\omega_{F}\right)$ where $F=P / Q$ is meromorphic. Away from the coordinate axes, $\omega_{F}$ can be written as

$$
\omega_{F}=\frac{\frac{1}{(2 \pi i)^{d}} \cdot \frac{P(\mathbf{x})}{x_{1} \ldots x_{d}} \exp (|\mathbf{r}| H(\mathbf{x}))}{Q(\mathbf{x})} d \mathbf{x}
$$

where the numerator and denominator are holomorphic functions. So wherever $Q_{d}$ and the $x_{j}$ do not vanish (for all $j$ ), we have

$$
\operatorname{Res}\left(\omega_{F}\right)=\frac{(-1)^{d-1}}{(2 \pi i)^{d}} \cdot \frac{P(\mathbf{x})}{x_{1} \ldots x_{d} Q_{d}(\mathbf{x})} e^{|\mathbf{r}| H(\mathbf{x})} d x_{1} \wedge \cdots \wedge d x_{d-1} .
$$


We now show existence and uniqueness of the residue form along the simple pole set $\mathcal{V}_{Q}$.

Proposition 2.6. Let $\eta$ be as in Definition 2.3. Then for any point $\mathbf{p} \in \mathcal{V}_{Q}$, there is a neighborhood $V \subseteq U$ of $\mathbf{p}$ and a holomorphic $(d-1)$-form $\theta$ on $V$ solving the equation

$$
d Q \wedge \theta=P d \mathbf{x}
$$

Furthermore, the restriction $\iota^{*} \theta$ induced by the inclusion $\iota: \mathcal{V}_{Q} \cap V \rightarrow V$ is unique.

Proof. First, we prove the existence of a solution $\theta$ to (2.3) in a neighborhood of $\mathbf{p}$. As $Q$ has a simple zero at $\mathbf{p}$, the implicit function theorem implies that for some neighborhood $V$ of $\mathbf{p}$ there is a biholomorphic function $\psi: \mathbb{C}^{d} \rightarrow V$ such that $Q(\psi(\mathbf{x}))=x_{1}$. Define the form $\theta_{0}$ by

$$
\theta_{0}=(P \circ \psi)|J| d x_{2} \wedge \cdots \wedge d x_{d},
$$

where $J$ is the Jacobian of the function $\psi$. The claim is that $\theta=\left(\psi^{-1}\right)^{*} \theta_{0}$ is a solution to $(2.3)$.

Indeed, by definition of $\theta_{0}$ we have that $d x_{1} \wedge \theta_{0}=(P \circ \psi)|J| d \mathbf{x}$. Pulling back both sides of this equation by $\psi^{-1}$ yields

$$
d\left(\psi^{-1}(\mathbf{x})_{1}\right) \wedge\left(\psi^{-1}\right)^{*} \theta_{0}=P \cdot\left(\psi^{-1}\right)^{*}(|J| d \mathbf{x}),
$$

which simplifies to $d Q \wedge \theta=P d \mathbf{x}$, as desired.

To prove uniqueness, assume that we have two $(d-1)$-forms $\theta$ and $\tilde{\theta}$ such that $d Q \wedge \theta=P d \mathbf{x}$ and $d Q \wedge \tilde{\theta}=P d \mathbf{x}$. Then $d Q \wedge(\theta-\tilde{\theta})=0$, which implies

$$
\psi^{*}(d Q \wedge(\theta-\tilde{\theta}))=d x_{1} \wedge \psi^{*}(\theta-\tilde{\theta})=0 .
$$

But this means that $\psi^{*}(\theta-\tilde{\theta})$ is a multiple of $d x_{1}$. Pulling back by $\left(\psi^{-1}\right)^{*}$, this implies that $\theta-\tilde{\theta}$ is a multiple of $d Q$. Finally, pulling back by $\iota^{*}$, this implies that $\iota^{*}(\theta-\tilde{\theta})$ is a multiple of $d(Q \circ \iota)=0$. Thus $\iota^{*}(\theta-\tilde{\theta})$ vanishes, and so $\iota^{*} \theta=\iota^{*} \tilde{\theta}$.

REMARK 2.7. Let $\eta$ be as in the definition of the residue form, and let $\psi: V \rightarrow$ $U$ be a biholomorphic function. Then

(1) The residue form is natural, i.e. $\operatorname{Res}(\eta)$ does not depend on the particular $P$ and $Q$ chosen to represent $\eta$ as $(P / Q) d \mathbf{x}$.

(2) The residue form is functorial, i.e. $\operatorname{Res}\left(\psi^{*} \eta\right)=\psi^{*} \operatorname{Res}(\eta)$ (where on the right side of the equation, $\psi$ is restricted to the domain $\left.\psi^{-1}\left(\mathcal{V}_{Q}\right)=\mathcal{V}_{Q \circ \psi}\right)$.

Theorem 2.8 (Cauchy-Leray Residue Theorem). Let $\eta$ be a meromorphic $d$ form on domain $U \subseteq \mathbb{C}^{d}$, with pole set $\mathcal{V} \subseteq U$ along which $\eta$ has only simple poles. Let $N$ be a d-chain in $U$, locally the product of a $(d-1)$-chain $C$ on $\mathcal{V}$ with a circle $\gamma$ in the normal slice to $\mathcal{V}$, oriented positively with respect to the complex structure of the normal slice. Then

$$
\int_{N} \eta=2 \pi i \int_{C} \operatorname{Res}(\eta)
$$

Proof. We proceed by examining the structure of the integral locally. So fix an arbitrary $\mathbf{p} \in C$. In a neighborhood $V \subseteq \mathbb{C}^{d}$ of $\mathbf{p}$, the surrounding space looks 
like a direct product of $\mathcal{V} \cap V$ (isomorphic to $\mathbb{C}^{d-1}$ for $V$ small) and the normal space to $\mathcal{V} \cap V$ (isomorphic to $\mathbb{C}$ ). Hence there is a biholomorphic function

$$
\begin{aligned}
\varphi: V & \rightarrow \mathbb{C} \times \mathbb{C}^{d-1} \\
\mathbf{x} & \mapsto\left(\varphi_{1}(\mathbf{x}), \varphi_{2}(\mathbf{x})\right)
\end{aligned}
$$

where the map $\varphi_{2}^{-1}$ is a parametrization of $\mathcal{V} \cap V$, and

$$
\begin{aligned}
& \varphi(\mathcal{V} \cap V)=\{0\} \times \varphi_{2}(\mathcal{V} \cap V), \\
& \varphi(N \cap V)=\gamma \times \varphi_{2}(C \cap V),
\end{aligned}
$$

where $\gamma \subseteq \mathbb{C}$ is a loop around the origin, positively oriented. Furthermore, if $V$ is chosen small enough, we can guarantee that the meromorphic form $\left(\varphi^{-1}\right)^{*} \eta$ has a global representation as $P / Q d \mathbf{x}$. Note that, by the structure of $\eta$ and definition of $\varphi, Q$ must vanish on the set

$$
\varphi(\mathcal{V} \cap V)=\left\{\mathbf{x} \in \mathbb{C}^{d}: x_{1}=0\right\}
$$

where it has only simple zeroes.

I claim that if we can prove the equality stated in the residue theorem restricted to $V$, we will be done with the theorem. This is due to the additivity of integration and the compactness of $C$ : we can split up a tubular neighborhood of $C$ (containing $N$ ) into finitely many such neighborhoods on which the theorem holds, then prove the theorem by breaking the integral into a sum over these pieces.

So without loss of generality, we may assume that this local structure holds globally on $C$ and that the domain of the map $\varphi$ is all of $\mathbb{C}^{d}$. By changing variables, we get

$$
\int_{N} \eta=\int_{\gamma \times \varphi_{2}(C)} \frac{P}{Q} d \mathbf{x}=\int_{\mathbf{p} \in \varphi_{2}(C)}\left(\int_{\gamma \times\{\mathbf{p}\}} \frac{P}{Q} d x_{1}\right) d x_{2} \wedge \cdots \wedge d x_{d}
$$

the upshot being the ability to split the above into an iterated integral, by the product structure of $\gamma \times \varphi_{2}(C)$.

The next step is to compute the inner integral from (2.4) by the ordinary residue theorem, but doing so will require a change of variables. To that end, define the function $\psi: \mathbb{C}^{d} \rightarrow \mathbb{C}^{d}$ by

$$
\psi(\mathbf{x})=\left(Q(\mathbf{x}), x_{2}, x_{3}, \ldots, x_{d}\right),
$$

and fix some $\mathbf{p} \in \mathbb{C}^{d-1}$. The claim is that $\psi$ is biholomorphic in a neighborhood $W \subseteq \mathbb{C}^{d}$ of $(0, \mathbf{p})$. By the inverse function theorem, this is true if and only if $|J(\mathbf{p})|=Q_{1}(\mathbf{p}) \neq 0$, where $J$ is the Jacobian of $\psi$. As $Q$ has a simple zero at $\mathbf{p}$, it can't be true that $Q_{i}(\mathbf{p})=0$ for all $i$. But $Q_{i}(\mathbf{p})=0$ for all $i \neq 1$, because $Q$ is constant (equal to 0 ) on the entire plane $x_{1}=0$. Thus $Q_{1}(\mathbf{p}) \neq 0$, as desired. Note that $\psi^{-1}$ must have the form

$$
\psi^{-1}(\mathbf{x})=\left(f(\mathbf{x}), x_{2}, x_{3}, \ldots, x_{d}\right)
$$

for some function $f$, and that $Q \circ \psi^{-1}=x_{1}$.

We'd like to perform a change of variables and compute the inner integral from (2.4) over the domain $\psi(\gamma \times\{\mathbf{p}\})$. The only problem with this is that there is no guarantee that $\gamma \times \varphi_{2}(C) \subseteq W$. But we can make this guarantee by shrinking $N$, i.e. shrinking the loop $\gamma$ closer to the origin, and by (potentially) restricting our attention to a small portion of $C$. Note that shrinking $N$ has no effect on the original integral (the new $N$ will differ from the old $N$ by a boundary, and we are 
integrating a closed form), and that, as we have already stated, we need only prove the residue theorem locally. Thus we may assume without loss of generality that $\gamma \times \varphi_{2}(C)$ is contained entirely within the domain of $\psi$.

After the suggested change of variables, we obtain

$$
\int_{N} \eta=\int_{\mathbf{p} \in \varphi_{2}(C)}\left(\int_{\psi(\gamma \times\{\mathbf{p}\})} \frac{P \circ \psi^{-1}}{x_{1}} \frac{\partial f}{\partial x_{1}} d x_{1}\right) d x_{2} \wedge \ldots \wedge d x_{n} .
$$

By the form of $\psi, \psi(\gamma \times\{\mathbf{p}\})$ is simply a loop around the origin in the plane $\left\{x \in \mathbb{C}^{d}:\left(x_{2}, \ldots, x_{d}\right)=\mathbf{p}\right\}$. So by the ordinary residue theorem we can compute

$$
\int_{\psi(\gamma \times\{\mathbf{p}\})} \frac{P \circ \psi^{-1}}{x_{1}} \frac{\partial f}{\partial x_{1}} d x_{1}=2 \pi i \cdot P\left(\psi^{-1}(0, \mathbf{p})\right) \frac{\partial f}{\partial x_{1}}(0, \mathbf{p}) .
$$

Substituting back into (2.4) yields

$$
\begin{aligned}
\int_{N} \eta & =2 \pi i \int_{\mathbf{p} \in \varphi_{2}(C)} P\left(\psi^{-1}(0, \mathbf{p})\right) \frac{\partial f}{\partial x_{1}}(0, \mathbf{p}) d x_{2} \wedge \ldots \wedge d x_{n} \\
& =2 \pi i \int_{\{0\} \times \varphi_{2}(C)} \operatorname{Res}\left(\frac{P \circ \psi^{-1} \cdot \frac{\partial f}{\partial x_{1}}}{x_{1}} d \mathbf{x}\right),
\end{aligned}
$$

where the second equality comes from the residue computation of Example 2.4.

But note that

$$
\begin{aligned}
\left(\psi^{-1}\right)^{*}\left(\frac{P}{Q} d \mathbf{x}\right) & =\frac{P \circ \psi^{-1}}{x_{1}}\left(\sum_{j=1}^{d} \frac{\partial f}{\partial x_{j}} d x_{j}\right) \wedge d x_{2} \wedge \cdots \wedge d x_{d} \\
& =\frac{P \circ \psi^{-1}}{x_{1}} \frac{\partial f}{\partial x_{1}} d \mathbf{x}
\end{aligned}
$$

and so the integral equation becomes

$$
\begin{aligned}
\int_{N} \eta & =2 \pi i \int_{\{0\} \times \varphi_{2}(C)} \operatorname{Res}\left(\left(\psi^{-1}\right)^{*}\left(\frac{P}{Q} d \mathbf{x}\right)\right) \\
& =2 \pi i \int_{\{0\} \times \varphi_{2}(C)} \operatorname{Res}\left(\left(\psi^{-1}\right)^{*}\left(\varphi^{-1}\right)^{*} \eta\right) .
\end{aligned}
$$

Finally, by the functoriality of the residue form, we obtain

$$
\int_{N} \eta=2 \pi i \int_{\{0\} \times \varphi_{2}(C)}\left(\psi^{-1}\right)^{*}\left(\varphi^{-1}\right)^{*} \operatorname{Res}(\eta)=2 \pi i \int_{C} \operatorname{Res}(\eta) .
$$

The residue theorem applies directly to the coefficient analysis of the previous subsection by the following corollary.

COROLlary 2.9. Under the assumptions and notation of Theorem 2.2

$$
a_{\mathbf{r}}=2 \pi i \int_{C} \operatorname{Res}\left(\omega_{F}\right)+\int_{T_{1}} \omega_{F},
$$

given the proper orientation of $C$.

Proof. By the residue theorem, $\int_{\partial \nu} \omega_{F}=2 \pi i \int_{C} \operatorname{Res}\left(\omega_{F}\right)$. The result follows by substituting this equality into the conclusion of Theorem 2.2. 
And thus the asymptotic coefficient analysis reduces to the integration of a $d-1$ form along a cycle on the pole set of the coefficient generating function. The final step is to compute this integral by means of the saddle point method.

2.3. Critical Points of the Height Function. The goal is to obtain an asymptotic expansion for $2 \pi i \int_{C} \operatorname{Res}\left(\omega_{F}\right)$, where $F=P / Q$ for some entire functions $P$ and $Q, F$ is analytic in a neighborhood of the origin, and $\mathcal{V}_{Q}$ is smooth. By Example 2.5 we can expect $\operatorname{Res}\left(\omega_{F}\right)$ to take the form

$$
\operatorname{Res}\left(\omega_{F}\right)=\frac{(-1)^{d-1}}{(2 \pi i)^{d}} \cdot \frac{P(\mathbf{x})}{x_{1} \ldots x_{d} Q_{d}(\mathbf{x})} e^{|\mathbf{r}| H(\mathbf{x})} d x_{1} \wedge \cdots \wedge d x_{d-1}
$$

(where $Q_{d}$ does not vanish), and as before we see that the exponential growth of this form is governed by the height function $h$. This motivates a deformation of the cycle $C$ along $\mathcal{V}_{Q}$, pushing $C$ down to a homologous cycle $\tilde{C}$ on which the maximum modulus of $h$ is minimized. This procedure is obstructed when the cycle gets trapped on a saddle point of $h$ on $\mathcal{V}_{Q}$, and the idea is to arrange $\tilde{C}$ so that the local maxima of $h$ along $\tilde{C}$ are all achieved at such saddle points. Away from the highest saddle points (the contributing points) the integral will contribute asymptotically negligible quantities, and near the contributing points the integral will be amenable to the saddle point method.

Thus the first task is to identify the location of the critical points of $h_{\hat{\mathbf{r}}} \mid \mathcal{V}_{Q}$. These points can be realized as the zero set of $d$ equations, as exhibited below.

Theorem 2.10 (Location of Critical Points). Assume $\hat{r}_{d} \neq 0$. Then the critical points of $h_{\hat{\mathbf{r}}}$ restricted to $\mathcal{V}_{Q}$ are precisely those points $\mathbf{p} \in \mathbb{C}^{d}$ satisfying the following d equations:

$$
\begin{aligned}
Q(\mathbf{p}) & =0, \\
\hat{r}_{d} p_{j} Q_{j}(\mathbf{p})-\hat{r}_{j} p_{d} Q_{d}(\mathbf{p}) & =0 \quad \forall j \neq d .
\end{aligned}
$$

In the case $d=2$, these critical points are actually saddle points of $h_{\hat{\mathbf{r}}} \mid \mathcal{V}_{Q}$.

For the purposes of computation it should be noted that when $Q$ is a polynomial, the above set of critical points is generically finite and can be found algorithmically by the method of Gröbner bases (see [CLO05, Section 1.3]).

Proof. The equation $Q(\mathbf{p})=0$ is clear: any critical point of $\left.h\right|_{\mathcal{V}_{Q}}$ will have to be on $\mathcal{V}_{Q}$. So we turn to the remaining $d-1$ equations.

Fix a point $\mathbf{p} \in \mathcal{V}_{Q}$ (not on the coordinate axes). By the Cauchy-Riemann equations, $\mathbf{p}$ is a critical point of $\Re\left(\left.H\right|_{\mathcal{V}_{Q}}\right)$ if and only if it is a critical point of $\Im\left(\left.H\right|_{\mathcal{V}_{Q}}\right)$. Thus $\mathbf{p}$ is a critical point of $\left.h\right|_{\mathcal{V}_{Q}}$ exactly when

$$
\nabla\left(\left.H\right|_{\mathcal{V}_{Q}}\right)(\mathbf{p})=0
$$

But $\nabla\left(\left.H\right|_{\mathcal{V}_{Q}}\right)(\mathbf{p})$ is simply the projection of $\nabla H(\mathbf{p})$ onto the tangent space $T_{\mathbf{p}} \mathcal{V}_{Q}$. Hence the previous equation is true if and only if

$$
\nabla H(\mathbf{p}) \| \nabla Q(\mathbf{p}),
$$

as $\nabla Q(\mathbf{p})$ is a vector normal to the tangent space to $\mathcal{V}_{Q}$ at $\mathbf{p}$. This condition reduces to the equation

$$
\left(\frac{-\hat{r}_{1}}{p_{1}}, \ldots, \frac{-\hat{r}_{d}}{p_{d}}\right)=\lambda\left(Q_{1}(\mathbf{p}), \ldots, Q_{d}(\mathbf{p})\right)
$$

for some scalar $\lambda$, which is captured by the remaining $d-1$ equations of the theorem. 
For the $d=2$ case, let $\mathbf{p}$ be any critical point of $\left.h\right|_{\mathcal{V}_{Q}}$ (hence a critical point of $\left.H\right|_{\mathcal{V}_{Q}}$ by the above). In a chart map in a neighborhood of the origin, we can write

$$
\left.H\right|_{\mathcal{V}_{Q}}(z)=c_{0}+c_{k} z^{k}(1+O(z)),
$$

for some constants $c_{0}$ and $c_{k}$ and $k \geq 2$. As $h=\Re(H)$, it follows that $\left.h\right|_{\mathcal{V}_{Q}}$ has a $k^{\text {th }}$ order saddle at $\mathbf{p}$.

After deforming the domain of integration so that $h$ is locally maximized at the critical points located above, the final step is to obtain an asymptotic expansion by applying the saddle point method near these points. In the case where $d=2$, this results in a single variable saddle integral. Specifically, we will make use of the following theorem.

THEOREM 2.11. Let $A$ and $\phi$ be holomorphic functions on a neighborhood of $0 \in \mathbb{C}$, with

$$
A(z)=\sum_{j=l}^{\infty} b_{j} z^{j}, \quad \phi(z)=\sum_{j=k}^{\infty} c_{j} z^{j}
$$

where $l \geq 0, k \geq 2$ and $b_{l} \neq 0, c_{j} \neq 0$. Let $\gamma:[-\varepsilon, \varepsilon] \rightarrow \mathbb{C}$ be any smooth curve with $\gamma(0)=0, \gamma^{\prime}(0) \neq 0$ and assume that $\Re \phi(\gamma(t)) \geq 0$ with equality only at $t=0$. Denote by $\gamma^{+}$the image of $\gamma$ restricted to the domain $[0, \varepsilon]$. Then for some coefficients $a_{j}$ we have a full asymptotic expansion

$$
\int_{\gamma^{+}} A(z) e^{-\lambda \phi(z)} d z=\sum_{j=l}^{\infty} \frac{a_{j}}{k} \Gamma\left(\frac{1+j}{k}\right)\left(c_{k} \lambda\right)^{-(1+j) / k}
$$

as $\lambda \rightarrow \infty$, where the choice of $k^{\text {th }}$ root in $\left(c_{k} \lambda\right)^{-(1+j) / k}$ is made by taking the principal root of $v^{-1}\left(c_{k} \lambda v^{k}\right)^{1 / k}$ where $v=\gamma^{\prime}(0)$. The leading two coefficients $a_{j}$ are given by

$$
a_{l}=b_{l}, \quad a_{l+1}=b_{l+1}-\frac{2+l}{k} \cdot \frac{c_{k+1}}{c_{k}} .
$$

For the purposes of computation it should be noted that each coefficient $a_{j}$ can be effectively computed from the values $b_{l}, \ldots, b_{j}$ and $c_{k}, \ldots, c_{k+j-l}$.

See $[$ Pem09] for the proof, or [Hen91, Section 11.8] for a treatment from which the above may be derived. It should be noted that, while the saddle point method is a very well known and well understood technique, it is often presented only as a method for solving a general class of problems - theorems are usually only given for limited, special case applications. Theorem 2.11 is stated in a generality not easily found in the literature.

\section{Application to Bicolored Supertrees}

The purpose of this section is to apply the methods of Section 2 to the example presented in the introduction. Throughout this section we use the notation of Section 2, and in the case of bicolored supertrees this means

$$
\begin{aligned}
F & =\frac{P}{Q}, P \text { and } Q \text { defined as in }(1.1) \\
|\mathbf{r}| & =n, \hat{\mathbf{r}}=\hat{\mathbf{r}}_{0}=(1,1) \\
H(x, y) & =-\ln x-\ln y \\
h(x, y) & =-\ln |x|-\ln |y| .
\end{aligned}
$$


Then as outlined in Section 2, the procedure will be as follows.

(1) Reduce the asymptotic computation to an integral on the variety $\mathcal{V}_{Q}$ using Corollary 2.9.

(2) Locate the critical points of $\left.h\right|_{\mathcal{V}_{Q}}$ and deform the contour of integration so as to minimize the maximum of $h$ at such points.

(3) Compute an asymptotic expansion for this integral by applying Theorem 2.11 near these maxima and bounding the order away from these maxima.

These three steps will be carried out in the subsections that follow. Thanks to all the work laid out in the previous section, many of these steps will be automatic. The most difficult step will be step (2), finding the new saddle point contour and actually proving that it possesses the right properties (Lemma 3.6). The rest will be a matter of applying the theorems when appropriate.

Before jumping into computations, however, we will need to do some initial work on describing the variety $\mathcal{V}_{Q}$.

3.1. Describing the Variety. Because $Q$ is quadratic in the variable $y$, we can explicitly solve $Q=0$ for $y$ as a function of $x$. This will allow us to parametrize $\mathcal{V}_{Q}$ by $x$ where possible. So define

$$
\begin{aligned}
& y_{1}(x)=\frac{-x^{2}+x^{3}-2+\sqrt{x^{4}+4 x^{2}-4 x^{3}+4}}{x^{5}}, \\
& y_{2}(x)=\frac{-x^{2}+x^{3}-2-\sqrt{x^{4}+4 x^{2}-4 x^{3}+4}}{x^{5}},
\end{aligned}
$$

where in each case the principal root is chosen. Then by the quadratic formula

$$
\mathcal{V}_{Q}=\left\{\left(x, y_{j}(x)\right): x \in \mathbb{C} \backslash\{0\}, j=1,2\right\} \cup\{(0,1 / 2)\},
$$

(though note that we may write $(0,1 / 2)=\left(0, y_{1}(0)\right)$ by analytically continuing $y_{1}$ to $x=0)$. To parametrize $\mathcal{V}_{Q}$ by $x$, we define the parametrization functions

$$
\iota_{1}(x)=\left(x, y_{1}(x)\right), \iota_{2}(x)=\left(x, y_{2}(x)\right) .
$$

For the purposes of later computation, it will be nice to know a domain on which these parametrization functions are holomorphic.

LEMMA 3.1. $\iota_{1}$ and $\iota_{2}$ are holomorphic on the punctured strip

$$
\{x \in \mathbb{C} \backslash 0: \Im(1 \pm \sqrt{1-2 i})<\Im x<\Im(1 \pm \sqrt{1+2 i})\} .
$$

Proof. By definition of the functions $y_{1}$ and $y_{2}$, the only points where $\iota_{1}$ and $\iota_{2}$ may fail to be holomorphic are when $x=0$ or $f(x)=x^{4}+4 x^{2}-4 x^{3}+4 \leq 0$ (by the choice of principal square root). Thus we examine when $f(x)$ is a nonpositive real number.

Denote $a=\Re(x)$ and $b=\Im(x)$. We are interested in when $f(a+i b) \leq 0$, so we first examine the equation $\Im f(a+i b)=0$, or

$$
4 b(a-1)\left(a^{2}-2 a-b^{2}\right)=0 .
$$

The solution set of the above equation is the union of the lines $a=1, b=0$ and the hyperbola $a^{2}-2 a-b^{2}=0$. The points $x=1 \pm \sqrt{1 \pm 2 i}$ where $f(x)=0$ partition the set $\Im f(x)=0$ into 5 components on which $\Re f(x)$ is either all positive or all negative (by continuity of $f$ on the connected set $\Im f(x)=0$ ). See Figure 1 , page 13 (dashed lines denoting the boundary of the strip). 


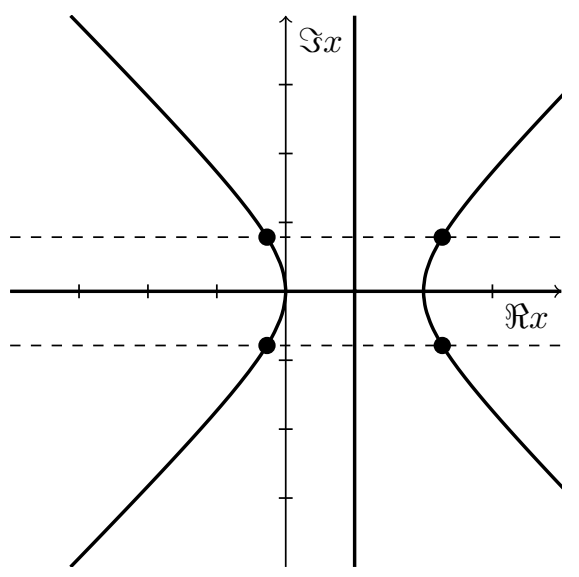

FiguRE 1. Zero sets of $\Im f$ and $f$.

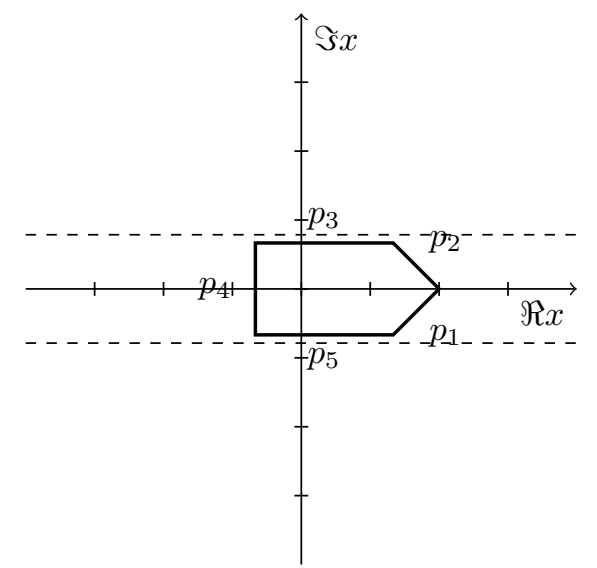

Figure 2. The pentagonal path $p$.

Only one of these 5 components enters the strip $\Im(1 \pm \sqrt{1-2 i})<\Im x<$ $\Im(1 \pm \sqrt{1+2 i}$ ), and by plugging a sample point of this component into $f$ (say $f(0)=4$ ) we see that $f$ is positive on this component. Thus at no point within this strip is $f(x) \leq 0$, and so $\iota_{1}$ and $\iota_{2}$ are holomorphic on the claimed domain.

Finally, as evidenced by Example 2.5, it will be useful for representing the residue form to know where $Q_{y}=\frac{\partial Q}{\partial y}$ is nonzero along $\mathcal{V}_{Q}$. Computing a Gröbner basis of the ideal $\left\langle Q, Q_{y}\right\rangle$ in Maple ([Wat08]) via the command

$\operatorname{Basis}([\mathrm{Q}, \operatorname{diff}(\mathrm{Q}, \mathrm{y})], \operatorname{plex}(\mathrm{y}, \mathrm{x}))$;

we obtain the univariate polynomial $x^{4}+4 x^{2}-4 x^{3}+4$ as the first basis element. Hence the $x$ coordinate of any point where $Q$ and $Q_{y}$ simultaneously vanish must be a root of this polynomial. This justifies the following remark.

REMARK 3.2. Along $\mathcal{V}_{Q}, Q_{y}$ is nonzero whenever $x \neq 1 \pm \sqrt{1 \pm 2 i}$ (the roots of the equation $\left.x^{4}+4 x^{2}-4 x^{3}+4=0\right)$.

3.2. Integral on the Pole Variety. The following lemma accounts for the first step of the analysis: using Corollary 2.9 to reduce the computation of $a_{n, n}$ to an integral on $\mathcal{V}_{Q}$.

Lemma 3.3. For $\varepsilon>0$, define

$$
C_{\varepsilon}=\{x \in \mathbb{C}:|x|=\varepsilon\},
$$

the circle of radius $\varepsilon$ about $0 \in \mathbb{C}$, oriented counterclockwise. Then for sufficiently small $\varepsilon>0$,

$$
a_{n, n}=2 \pi i \int_{\iota_{1}\left(C_{\varepsilon}\right)} \operatorname{Res}\left(\omega_{F}\right)+2 \pi i \int_{\iota_{2}\left(C_{\varepsilon}\right)} \operatorname{Res}\left(\omega_{F}\right) .
$$

ProOf. We first verify that the variety $\mathcal{V}_{Q}$ is smooth. This is true only if $Q$, $Q_{x}$ and $Q_{y}$ do not simultaneously vanish, which is true if and only if the variety $I=$ $\left\langle Q, Q_{x}, Q_{y}\right\rangle$ is trivial (the whole polynomial ring). We check this algorithmically, using Gröbner bases. In Maple, we compute the Gröbner basis of $I$ with the command 
$\operatorname{Basis}([Q, \operatorname{diff}(Q, x), \operatorname{diff}(Q, y)], \operatorname{plex}(y, x))$

Maple returns the basis [1] for $I$, so the ideal is indeed trivial.

Now, let $\varepsilon>0, \delta>0$ be sufficiently small so that

$$
a_{n, n}=\int_{T_{0}} \omega_{F}, \quad \text { where } T_{0}=\left\{(x, y) \in \mathbb{C}^{2}:|x|=\varepsilon,|y|=\delta\right\}
$$

by Cauchy's Integral Formula. Define the quantities

$$
\begin{aligned}
& m_{0}=\inf \left\{\left|y_{j}(x)\right|: x \in C_{\varepsilon}, j=1,2\right\}, \\
& M_{0}=\sup \left\{\left|y_{j}(x)\right|: x \in C_{\varepsilon}, j=1,2\right\} .
\end{aligned}
$$

For $\varepsilon$ sufficiently small, note that $M_{0}<\infty$ (by continuity of the $y_{j}$; see Lemma 3.1) and $m_{0}>0$ (the $x$-axis intersects $\mathcal{V}_{Q}$ only at the point $(2,0)$ ).

Assume $\delta$ is chosen small enough so that $\delta<m_{0}$. Fix any $M>M_{0}$. Then define the homotopy

$$
\begin{aligned}
K: T_{0} \times[0,1] & \rightarrow \mathbb{C}^{2} \\
(x, y, t) & \mapsto\left(x, y\left(1+t\left(\frac{M}{\delta}-1\right)\right)\right),
\end{aligned}
$$

expanding $T_{0}$ in the $y$ direction past $\mathcal{V}_{Q}$. Then $K$ intersects $\mathcal{V}_{Q}$ in the set $C=$ $\iota_{1}\left(C_{\varepsilon}\right) \cup \iota_{2}\left(C_{\varepsilon}\right)$ and avoids the coordinate axes. Furthermore, $K$ intersects $\mathcal{V}_{Q}$ transversely (as $K$ expands in the $y$ direction, intersecting $\mathcal{V}_{Q}$ where it is a graph of $x$ ). Thus, by Corollary 2.9 we obtain

$$
a_{n, n}=2 \pi i \int_{\iota_{1}\left(C_{\varepsilon}\right)} \operatorname{Res}\left(\omega_{F}\right)+2 \pi i \int_{\iota_{2}\left(C_{\varepsilon}\right)} \operatorname{Res}\left(\omega_{F}\right)+\int_{T_{1}} \omega_{F},
$$

where $C_{\varepsilon}$ is oriented counterclockwise (determined by examination of Theorem 2.2 and the Residue Theorem).

Now fix $n$ large and let $M$ vary. As the rest of the terms in (3.2) have no $M$ dependence, $\int_{T_{1}} \omega_{F}$ must be a constant function of $M$. But by trivial bounds, we can show that

$$
\int_{T_{1}} \omega_{F}=O\left(M^{1-n}\right) \text { as } M \rightarrow \infty,
$$

as $\frac{P}{(2 \pi i)^{2} x y Q}=O(1), \exp (n H)=O\left(M^{-n}\right)$ and the area of $T_{1}$ is $O(M)$. For $n>1$, $M^{1-n} \rightarrow 0$ as $M \rightarrow \infty$. Hence the only constant $\int_{T_{1}} \omega_{F}$ can be equal to is 0 .

3.3. Saddle Points and Contour Deformation. Step (2) in the analysis is to locate the saddle points of $\left.h\right|_{\mathcal{V}_{Q}}$ and deform the contour of integration appropriately, using this information. The saddle points can be found automatically as follows.

LEMMA 3.4. $\left.h\right|_{\mathcal{V}_{Q}}$ has three saddle points, located at

$$
\begin{aligned}
\left(2, \frac{1}{8}\right) & =\iota_{1}(2), \\
\left(1-\sqrt{5}, \frac{3+\sqrt{5}}{16}\right) & =\iota_{1}(1-\sqrt{5}), \\
\left(1+\sqrt{5}, \frac{3-\sqrt{5}}{16}\right) & =\iota_{2}(1+\sqrt{5}) .
\end{aligned}
$$


Proof. By Theorem 2.10, the critical points of $\left.h\right|_{\mathcal{V}_{Q}}$ are those points where $Q$ and $x Q_{x}-y Q_{y}$ simultaneously vanish. We can compute these points algorithmically by computing the Gröbner basis for the ideal $I=\left\langle Q, x Q_{x}-y Q_{y}\right\rangle$. This is done in Maple with the command

$\operatorname{Basis}([Q, x * \operatorname{diff}(Q, x)-y * \operatorname{diff}(Q, y)], \operatorname{plex}(y, x))$;

which returns a basis consisting of the following two polynomials:

$$
32-8 x^{2}-32 x+20 x^{3}-8 x^{4}+x^{5}, \quad x^{4}-48-6 x^{3}+8 x^{2}+128 y+16 .
$$

The first polynomial factors as $\left(x^{2}-2 x-4\right)(x-2)^{3}$, with roots $x=2$ and $x=1 \pm \sqrt{5}$. Substituting these values of $x$ into the second polynomial and solving for $y$ yields the critical points claimed in the lemma.

We note here the interesting geometry near the critical point $(2,1 / 8)$, which will turn out to be the sole contributing point. Expanding $H\left(\iota_{1}(x)\right)$ near $x=2$, we obtain

$$
H\left(\iota_{1}(x)\right)=H\left(\iota_{1}(2)\right)+\frac{1}{16}(x-2)^{4}+O\left((x-2)^{6}\right),
$$

and hence $\left.h\right|_{\mathcal{V}_{Q}}$ has a degenerate saddle (of order 4) near this critical point, with steepest descent directions emanating from $x=2$ at angles $\pi / 4+j(\pi / 2)$ radians $(j=1,2,3,4$. $)$. We also see that along the path $|x|=2, h\left(\iota_{1}(x)\right)$ is locally minimized at $x=2$, as this path passes through the critical point along ascent directions. Hence $x=2$ is a local maximum for $\left|y_{1}(x)\right|$ along this path, and so there are points $(x, y) \in \mathcal{V}_{Q}$ near $(2,1 / 8)$ such that $|x|=2$ and $|y|<1 / 8$. Because $\mathcal{V}_{Q}$ cuts in toward the origin near $\iota_{1}(2)$, this critical point is not on the boundary of the domain of convergence of $F$. In the terminology of the introduction, this critical point is not minimal.

Knowing where the saddle points of $h$ are, the next task is to deform the contour of integration in (3.1) so as to minimize the maximum modulus of $h$ along the new contour at said saddle points. The integral over domain $\iota_{2}\left(C_{\varepsilon}\right)$ will actually be shown to vanish, while the domain $\iota_{1}\left(C_{\varepsilon}\right)$ will be pushed to a "pentagonal" path through the critical point $(2,1 / 8)$.

The specific path to which $\iota_{1}\left(C_{\varepsilon}\right)$ will be deformed is $\iota_{1}(p)$ where $p$ is the pentagonal path depicted in Figure 2 on page 13, with vertices at the points

$$
\left\{\frac{4}{3}-i \frac{2}{3}, 2, \frac{4}{3}+i \frac{2}{3},-\frac{2}{3}+i \frac{2}{3},-\frac{2}{3}-i \frac{2}{3}\right\} \text {. }
$$

Denote by $p_{1}, \ldots, p_{5}$ the edges of $p$, as denoted in the figure.

Performing the suggested deformation results in the following lemma.

LEMMA 3.5.

$$
a_{n, n}=2 \pi i \int_{\iota_{1}(p)} \operatorname{Res}\left(\omega_{F}\right),
$$

where $p$ is oriented counterclockwise.

Proof. For $\delta<\varepsilon$, let $K$ be a homotopy shrinking the circle $C_{\varepsilon}$ to the circle $C_{\delta}$. By holomorphicity of $\iota_{2}$ (Lemma 3.1), $\iota_{2} \circ K$ is a homotopy from $\iota_{2}\left(C_{\varepsilon}\right)$ to $\iota_{2}\left(C_{\delta}\right)$ along $\mathcal{V}_{Q}$, and $\operatorname{Res}\left(\omega_{F}\right)$ is holomorphic along this homotopy. By Stokes' Theorem we obtain

$$
\int_{\iota_{2}\left(C_{\varepsilon}\right)} \operatorname{Res}\left(\omega_{F}\right)=\int_{\iota_{2}\left(C_{\delta}\right)} \operatorname{Res}\left(\omega_{F}\right) .
$$


Now fix $n$ large and let $\delta$ vary. Note that as the left hand side of the above equation has no $\delta$ dependence, neither does the right.

By the fact that $y_{2}(x)=-4 x^{-5}(1+O(x))$ as $x \rightarrow 0$, we get that $\frac{-P}{(2 \pi i)^{2} x y Q y}=$ $O\left(\delta^{-4}\right), \exp (n H)=O\left(\delta^{4 n}\right)$ and the area of $\iota_{2}\left(C_{\delta}\right)$ is $O\left(\delta^{-4}\right)$ as $\delta \rightarrow 0$. This implies that

$$
\int_{\iota_{2}\left(C_{\delta}\right)} \operatorname{Res}\left(\omega_{F}\right)=\int_{\iota_{2}\left(C_{\delta}\right)} \frac{1}{(2 \pi i)^{2}} \cdot \frac{-P}{x y Q_{y}} e^{n H} d x=O\left(\delta^{4 n-8}\right)
$$

as $\delta \rightarrow 0$ (note that this representation of the residue is valid by Remark 3.2). For $n>2, \delta^{4 n-8} \rightarrow 0$ as $\delta \rightarrow 0$. Thus we must have that this integral is equal to 0 .

As for the the integral over $\iota_{1}\left(C_{\varepsilon}\right)$ in (3.1), let $K$ now be a homotopy expanding the circle $C_{\varepsilon}$ to the pentagonal path $p$. Then by Lemma $3.1, \iota_{1} \circ K$ is a homotopy from $\iota_{1}\left(C_{\varepsilon}\right)$ to $\iota_{2}(p)$ along $\mathcal{V}_{Q}$, and $\operatorname{Res}\left(\omega_{F}\right)$ is likewise holomorphic along the image of this homotopy. Then by Stokes' Theorem,

$$
\int_{\iota_{1}\left(C_{\varepsilon}\right)} \operatorname{Res}\left(\omega_{F}\right)=\int_{\iota_{1}(p)} \operatorname{Res}\left(\omega_{F}\right)
$$

where $p$ is oriented counterclockwise. The theorem follows.

Now we show that $h$ is indeed maximized on $\iota_{1}(p)$ uniquely at the point $(2,1 / 8)$. That this is true local to the saddle point $(2,1 / 8)$ is clear from the form of $H$ near this point, as explored following the proof of Lemma 3.4. To show that this is true globally will require more effort.

LEMMA 3.6. $h\left(\iota_{1}(x)\right)<h\left(\iota_{1}(2)\right)=\ln 4 \quad \forall x \in p \backslash\{2\}$.

Proof. Because $h\left(\iota_{1}(x)\right)$ is continuous on the connected set $p$, we need only show that $h\left(\iota_{1}(x)\right) \neq \ln 4$ for all $x \in p \backslash\{2\}$, and that $h\left(\iota_{1}(x)\right)<\ln 4$ for some $x \in p \backslash$ $\{2\}$. The latter condition can be easily checked by plugging some arbitrary point into $h\left(\iota_{1}(x)\right)$. As for the former condition, the idea will be to cook up some polynomial equations that must be satisfied in order for it to be true that $h\left(\iota_{1}(x)\right)=\ln 4$. We then use techniques from computational algebra to show that these equations can not be satisfied for any $(x, y)$ with $x \in p \backslash\{2\}$ and $y=y_{1}(x)$. follows:

The conditions from which we will derive our polynomial equations are as

(1) $x \in p_{j}$ for some $j \in\{1, \ldots, 5\}$.

(2) $y$ such that $(x, y) \in \mathcal{V}_{Q}$.

(3) $h(x, y)=\ln 4$, or $e^{h(x, y)}=4$.

Each of these conditions implies a (set of) polynomial equations in the variables $\Re(x), \Im(x), \Re(y)$ and $\Im(y)$, as we will show shortly. Note that we are throwing away some important information in condition 2 above, namely we want $y=y_{1}(x)$, not $y=y_{2}(x)$. This will be important later in the proof.

We examine first the case where $x \in p_{3}$. Denote $a=\Re(x), b=\Im(x), c=\Re(y)$ and $d=\Im(y)$. Then condition 1 implies the polynomial constraint:

$$
P_{1}=b-\frac{2}{3}=0 \text {. }
$$

Note: condition 1 implies the additional constraint $a \in[-2 / 3,4 / 3]$, which we will make use of shortly. 
Condition 2 implies the following two polynomial constraints:

$$
\begin{aligned}
& P_{2}=\Re(Q(a+i b, c+i d))=0, \\
& P_{3}=\Im(Q(a+i b, c+i d))=0 .
\end{aligned}
$$

Finally, condition 3 translates to $4|x||y|=1$, or

$$
P_{4}=16\left(a^{2}+b^{2}\right)\left(c^{2}+d^{2}\right)-1=0 .
$$

We are interested in whether these four polynomial equations have a common real-valued solution, and we will use Gröbner bases and Sturm sequences to answer this question. Since we expect the variety generated by $I=\left\langle P_{1}, P_{2}, P_{3}, P_{4}\right\rangle$ to be finite $-I$ is generated by four polynomials in four unknowns - we hope to use Gröbner bases to eliminate variables and produce a univariate polynomial $B(a) \in I$. Any point $(a, b, c, d)$ solving $P_{j}=0$ for all $j$ will likewise solve $B=0$. Then we try to use Sturm sequences to that such a $B$ has no real roots $a \in[-2 / 3,4 / 3]$, proving that $h\left(\iota_{1}(x)\right) \neq \ln 4$ for $x \in p_{3}$.

We compute the Gröbner basis with the command

$\operatorname{Basis}([\mathrm{P} 1, \mathrm{P} 2, \mathrm{P} 3, \mathrm{P} 4], \mathrm{plex}(\mathrm{d}, \mathrm{c}, \mathrm{b}, \mathrm{a}))$

and find that the first element $B$ of the basis is univariate in the variable $a$, a polynomial of degree 16 . We can check that $B(-2 / 3) \neq 0$ and $B(4 / 3) \neq 0$ by direct computation in Maple. To check whether or not $B$ has any roots on the interval $(-2 / 3,4 / 3)$ we employ Sturm's Theorem (see [BPR06, p. 52]).

To employ Sturm's Theorem, we must verify that $B$ is squarefree. This is true if and only if the ideal $\left\langle B, B^{\prime}\right\rangle$ is equal to the trivial ideal $\langle 1\rangle$. Indeed, computing the Gröbner basis for $\left\langle B, B^{\prime}\right\rangle$

$\operatorname{Basis}([B, \operatorname{diff}(B, a)], \operatorname{plex}(a))$;

returns the trivial basis [1], i.e. $B$ is squarefree.

Then to count the number of roots in $(-2 / 3,4 / 3)$ via Sturm's Theorem, we enter the command

$\operatorname{sturm}(\operatorname{sturmseq}(B, a), a,-2 / 3,4 / 3)$

and Maple returns that there are 0 real roots on the interval $(-2 / 3,4 / 3)$.

Computations are similar for $p_{4}$ and $p_{5}$, but things are a bit more complicated along $p_{1}$ and $p_{2}$. Let's look at $p_{2}$. The first polynomial equation becomes

$$
P_{1}=a+b-2=0,
$$

with $a \in[4 / 3,2]$, while the rest of the polynomial equations remain the same. Going through the same procedure as before, we can produce a Gröbner basis for $\left\langle P_{1}, P_{2}, P_{3}, P_{4}\right\rangle$ with an element $B(a)$ univariate in $a . B(a)$ factors as

$$
B(a)=(a-2)^{4} \tilde{B}(a),
$$

where by direct computation we see that $\tilde{B}$ is nonzero at $a=4 / 3$ and $a=2$. Note: we expected that $B$ would have a root at $a=2$, corresponding to the fact that $h\left(\iota_{1}(2)\right)=\ln 4$.

The next step would be to attempt to show that $\tilde{B}$ has no roots on the interval $(4 / 3,2)$, but this is not true. Using Sturm sequences, one can show that $\tilde{B}$ has exactly one root $a_{0} \in(4 / 3,2)$, and this is because there is a pair $x, y$ with $x \in p_{2} \backslash\{2\}$ 
and $h(x, y)=\ln 4$. The claim is that this corresponds to a point where $y=y_{2}(x)$, not where $y=y_{1}(x)$.

To see that there must be such a pair, note that $y_{2}(x) \rightarrow 0$ as $x \rightarrow 2$. Hence $h\left(\iota_{2}(x)\right) \rightarrow \infty$ as $x \rightarrow 2$. But by direct computation we can show that $h\left(\iota_{2}(4 / 3)\right)<$ $\ln 4$. As $h\left(\iota_{2}(x)\right)$ is continuous on $p_{2} \backslash\{2\}$, there must be some $x \in p_{2} \backslash\{2\}$ such that $h\left(\iota_{2}(x)\right)=\ln 4$. This pair $x, y=y_{2}(x)$ satisfies the polynomial equations $P_{j}=0$.

Now assume by way of contradiction that $h\left(\iota_{1}(x)\right)=\ln 4$ for some $x \in p_{2} \backslash\{2\}$. Because $\tilde{B}$ has just one root $a_{0} \in(4 / 3,2)$, it must be that this occurs at the same $x$ value for which $h\left(\iota_{2}(x)\right)=\ln 4$, specifically $x_{0}=a_{0}+\left(2-a_{0}\right) i$. Hence we have

$$
\left|x_{0}\right|\left|y_{1}\left(x_{0}\right)\right|=\left|x_{0}\right|\left|y_{2}\left(x_{0}\right)\right|=\frac{1}{4},
$$

which implies that $\left|y_{1}\right|=\left|y_{2}\right|$ at the point $x_{0}$. So at this value of $x$ we have

$$
c^{2}+d^{2}=|y|^{2}=\left|y_{1} y_{2}\right|=\frac{|x-2|}{|x|^{5}}
$$

The preceding equation implies that $|x|^{10}\left(c^{2}+d^{2}\right)^{2}=|x-2|^{2}$, which translates into the polynomial equation

$$
P_{5}=\left(a^{2}+b^{2}\right)^{5}\left(c^{2}+d^{2}\right)^{2}-\left((a-2)^{2}+b^{2}\right)=0 .
$$

We now have a new polynomial equation that must be satisfied in order that $h\left(\iota_{1}(x)\right)=\ln 4$ on $p_{2} \backslash\{2\}$. But if we compute a Gröbner basis for $\left\langle P_{1}, \ldots, P_{5}\right\rangle$, we get the trivial basis [1], meaning that the polynomials have no common solution. Hence $h\left(\iota_{1}(x)\right) \neq \ln 4$ for $x \in p_{2} \backslash\{2\}$. Analogous methods can be used to handle the case of $p_{1}$.

3.4. Saddle Point Integration. The final step in the analysis is to use saddle point techniques and order bounds to prove (1.2).

THEOREM 3.7.

$$
k_{n}=a_{n, n} \sim \frac{4^{n}}{8 \Gamma(3 / 4) n^{5 / 4}} .
$$

Proof. We proceed from Lemma 3.5. The theorem will be proved in 2 steps: bounding the integral in (3.3) outside a neighborhood of the critical point, then applying saddle point techniques near that critical point.

For any neighborhood $N$ of $x=2$, we look at $\int_{\iota_{1}(p \backslash N)} \operatorname{Res}\left(\omega_{F}\right)$, which can be written as

$$
\int_{\iota_{1}(p \backslash N)} \frac{1}{(2 \pi i)^{2}} \cdot \frac{-P}{x y Q_{y}} e^{n H} d x
$$

(note that this representation is valid by Remark 3.2). As $h \circ \iota_{1}$ is continuous on the compact set $p \backslash N, h \circ \iota_{1}$ achieves an upper bound $M$ on $p \backslash N$. By Lemma 3.6, $M<\ln 4$. Thus by trivial bounds we have

$$
\int_{\iota_{1}(p \backslash N)} \operatorname{Res}\left(\omega_{F}\right)=O\left(e^{M n}\right)=o\left((4-\delta)^{n}\right)
$$

for sufficiently small $\delta>0$, as $n \rightarrow \infty$. Hence

$$
a_{n, n}=2 \pi i \int_{\iota_{1}(p \cap N)} \operatorname{Res}\left(\omega_{F}\right)+o\left((4-\delta)^{n}\right) .
$$

for any neighborhood $N$ of $x=2$, provided $\delta$ is sufficiently small. 
For $N$ small enough, $p \cap N=\left(p_{1} \cap N\right) \cup\left(p_{2} \cap N\right)$. We examine the integral over $\iota_{1}\left(p_{1} \cap N\right)$ and $\iota_{1}\left(p_{2} \cap N\right)$ separately, starting with $\iota_{1}\left(p_{2} \cap N\right)$. By using the aforementioned representation of the residue form (and changing variables), we obtain

$$
2 \pi i \int_{\iota_{1}\left(p_{2} \cap N\right)} \operatorname{Res}\left(\omega_{F}\right)=\int_{p_{2} \cap N} \frac{1}{2 \pi i} \cdot \frac{-P\left(\iota_{1}(x)\right)}{x y_{1}(x) Q_{y}\left(\iota_{1}(x)\right)} e^{n H\left(\iota_{1}(x)\right)} d x .
$$

After another change of variables $(x \rightarrow x+2)$ and a suitable choice of neighborhood $N$, the above integral can be rewritten as

$$
4^{n} \int_{\gamma^{+}} A(x) e^{-n \phi(x)} d x
$$

where we have, for some fixed $\varepsilon>0$,

$$
\begin{aligned}
& \gamma(x)=(i-1) x ; x \in[-\varepsilon, \varepsilon], \\
& A(x)=\frac{1}{2 \pi i} \cdot \frac{-P\left(\iota_{1}(x+2)\right)}{(x+2) y_{1}(x+2) Q_{y}\left(\iota_{1}(x+2)\right)}, \\
& \phi(x)=\ln 4-H\left(\iota_{1}(x+2)\right),
\end{aligned}
$$

and we recall that $\gamma^{+}$is the restriction of the image of $\gamma$ to the domain $[0, \varepsilon]$. The series expansion of $A$ and $\phi$ at $x=0$ begin

$$
\begin{aligned}
& A(x)=\frac{i}{16 \pi} x^{3}+\frac{i}{32 \pi} x^{4}+O\left(x^{5}\right), \\
& \phi(x)=\frac{-1}{16} x^{4}+O\left(x^{6}\right),
\end{aligned}
$$

and $\Re \phi(x)$ is uniquely minimized on $\gamma^{+}$at $x=0$ where we have $\phi(0)=0$, as a consequence of Lemma 3.6. Thus this is exactly the situation where the saddle point technique of Theorem 2.11 can be applied. The values of $b_{j}$ and $c_{j}$ are as in the expansions above. Then $v=\gamma^{\prime}(0)=i-1$, and we compute the principal root

$$
\frac{\left(c_{k} n v^{k}\right)^{1 / k}}{v}=\frac{\left((-1 / 16) n(i-1)^{4}\right)^{1 / 4}}{i-1}=\frac{-1-i}{2 \sqrt{2}} n^{1 / 4}
$$

The conclusion of Theorem 2.11 is then

$$
2 \pi i \int_{\iota_{1}\left(p_{2} \cap N\right)} \operatorname{Res}\left(\omega_{F}\right)=4^{n}\left(\frac{-i}{4 \pi} n^{-1}+\frac{(1+i) \sqrt{2} \Gamma(5 / 4)}{8 \pi} n^{-5 / 4}+O\left(n^{-3 / 2}\right)\right)
$$

As for the integral over $\iota_{1}\left(p_{1} \cap N\right)$, the same argument yields

$$
2 \pi i \int_{\iota_{1}\left(p_{1} \cap N\right)} \operatorname{Res}\left(\omega_{F}\right)=-4^{n} \int_{\gamma^{+}} A(x) e^{-n \phi(x)} d x
$$

where $A$ and $\phi$ are the same but $\gamma$ is defined by $\gamma(x)=(-i-1) x$ (and the negative sign out in front comes from a reversal of orientation). For $v=\gamma^{\prime}(0)=-i-1$, we compute the principal root

$$
\frac{\left(c_{k} n v^{k}\right)^{1 / k}}{v}=\frac{\left((-1 / 16) n(-i-1)^{4}\right)^{1 / 4}}{-i-1}=\frac{-1+i}{2 \sqrt{2}} n^{1 / 4} .
$$

Then by Theorem 2.11 we obtain

$$
2 \pi i \int_{\iota_{1}\left(p_{1} \cap N\right)} \operatorname{Res}\left(\omega_{F}\right)=4^{n}\left(\frac{i}{4 \pi} n^{-1}+\frac{(1-i) \sqrt{2} \Gamma(5 / 4)}{8 \pi} n^{-5 / 4}+O\left(n^{-3 / 2}\right)\right) .
$$


Adding up the contribution over each piece and plugging into (3.4) yields

$$
a_{n, n}=4^{n}\left(\frac{\sqrt{2} \Gamma(5 / 4)}{4 \pi} n^{-5 / 4}+O\left(n^{-3 / 2}\right)\right)+o\left((4-\delta)^{n}\right) \sim \frac{4^{n} \sqrt{2} \Gamma(5 / 4)}{4 \pi} n^{-5 / 4} .
$$

Using the identity $\Gamma(5 / 4) \Gamma(3 / 4)=\pi /(2 \sqrt{2})$, the theorem follows.

\section{References}

[AY83] Lev A. Aŭzenberg and Aleksandr P. Yuzhakov, Integral representations and residues in multidimensional complex analysis, Translations of Mathematical Monographs, vol. 58, American Mathematical Society, Providence, RI, 1983, Translated from the Russian by H. H. McFaden, Translation edited by Lev J. Leifman. MR MR735793 (85a:32006)

[BPR06] Saugata Basu, Richard Pollack, and Marie-Françoise Roy, Algorithms in real algebraic geometry, second ed., Algorithms and Computation in Mathematics, vol. 10, SpringerVerlag, Berlin, 2006. MR MR2248869 (2007b:14125)

[Bre93] Glen E. Bredon, Topology and geometry, Graduate Texts in Mathematics, vol. 139, Springer-Verlag, New York, 1993. MR MR1224675 (94d:55001)

[CLO05] David A. Cox, John Little, and Donal O'Shea, Using algebraic geometry, second ed., Graduate Texts in Mathematics, vol. 185, Springer, New York, 2005. MR MR2122859 (2005i:13037)

[FS09] Philippe Flajolet and Robert Sedgewick, Analytic combinatorics, Cambridge University Press, Cambridge, 2009. MR MR2483235

[Hen91] Peter Henrici, Applied and computational complex analysis. Vol. 2, Wiley Classics Library, John Wiley \& Sons Inc., New York, 1991, Special functions - integral transformsasymptotics - continued fractions, Reprint of the 1977 original, A Wiley-Interscience Publication. MR MR1164865 (93b:30001)

[Pem09] Robin Pemantle, Analytic combinatorics in more than one variable: Chapter 5, December 2009, http://www.math. upenn. edu/ pemantle/581-html/chapter05.pdf.

[PW02] Robin Pemantle and Mark C. Wilson, Asymptotics of multivariate sequences. I. Smooth points of the singular variety, J. Combin. Theory Ser. A 97 (2002), no. 1, 129-161. MR MR1879131 (2003a:05015)

[PW08] _ Twenty combinatorial examples of asymptotics derived from multivariate generating functions, SIAM Rev. 50 (2008), no. 2, 199-272. MR MR2403050 (2009b:05023)

[RW08] Alexander Raichev and Mark C. Wilson, A new approach to asymptotics of Maclaurin coefficients of algebraic functions, Report CDMTCS-322, Centre for Discrete Mathematics and Theoretical Computer Science, University of Auckland, New Zealand, April 2008, http://www.cs.auckland.ac.nz/CDMTCS/researchreports/322alexmcw.pdf.

[Saf00] Konstantin V. Safonov, On power series of algebraic and rational functions in $\mathbf{C}^{n}$, J. Math. Anal. Appl. 243 (2000), no. 2, 261-277. MR MR1741523 (2001f:32001)

[Sha92] Boris V. Shabat, Introduction to complex analysis. Part II, Translations of Mathematical Monographs, vol. 110, American Mathematical Society, Providence, RI, 1992, Functions of several variables, Translated from the third (1985) Russian edition by J. S. Joel. MR MR1192135 (93g:32001)

[Slo09] Neil J. A. Sloane (ed.), The on-line encyclopedia of integer sequences, no. A168506, 2009, http://www.research.att.com/ njas/sequences/A168506.

[Wat08] Waterloo Maple Inc., Maple, version 12, Waterloo, Ontario, 2008.

Department of Mathematics, 209 South 33rd Street, Philadelphia, PA 19104

E-mail address: tdevries@math.upenn.edu 